\title{
Mujeres académicas frente al techo de cristal. Un estudio de caso en educación superior
}

\section{Women academics facing the glass ceiling. A case study in higher education}

DOI: $10.46932 / \mathrm{sfjdv} 2 \mathrm{n} 2-056$

Received in: january 1st, 2020

Accepted in: March 30th, 2020

\author{
Mireya Martí Reyes \\ Doctora en Ciencias sobre Arte por el Instituto Superior de Arte, de la Habana, Cuba. \\ Universidad de Guanajuato, México \\ Lascuráin de Retana Núm. 5, Guanajuato, Gto., México \\ E-mail: mireya@ugto.mx

\section{Cirila Cervera Delgado} \\ Doctora en Historia por la Universidad Autónoma de Zacatecas, México. \\ Universidad de Guanajuato, México \\ Lascuráin de Retana Núm. 5, Guanajuato, Gto., México \\ E-mail: cirycervera@ugto.mx
}

\section{RESUMEN}

Dada la fuerte raíz y tradición patriarcal que prevalecen en nuestro tiempo y países, se ha normalizado la ausencia de mujeres en puestos de alta jerarquía en cualquier tipo de organizaciones. El conjunto de obstáculos que impiden a las mujeres acceder a los puestos del más alto nivel recibe el nombre de techo de cristal: un conjunto de barreras invisibles, que es necesario revelarlas. El techo de cristal está presente también en las instituciones de educación superior (IES), de allí que hayamos emprendido esta investigación con la finalidad de dar cuenta de las relaciones asimétricas entre académicas y académicos, tomando una de ellas como unidad de análisis particular. Los hallazgos informan que la cúpula de los organigramas la ocupan los hombres, cediendo a las mujeres puestos a partir del tercer nivel; de aquí que hacemos una descripción del techo de cristal en esta entidad, de acuerdo con el análisis documental realizado.

Palabras claves: Educación superior, Techo de vidrio, Suelo pegajoso, Género.

\begin{abstract}
Given the strong patriarchal roots and traditions that prevail in our times and countries, the absence of women in high hierarchical positions in any type of organization has become normalized. The set of obstacles that prevent women from accessing top-level positions is known as the glass ceiling: a set of invisible barriers that must be revealed. The glass ceiling is also present in higher education institutions (HEIs), which is why we undertook this research in order to account for the asymmetrical relationships between male and female academics, taking one of them as a particular unit of analysis. The findings show that the top of the organizational charts are occupied by men, giving way to women positions from the third level onwards; hence we make a description of the glass ceiling in this entity, according to the documentary analysis carried out.
\end{abstract}

Key words: Higher education, Glass ceiling, Sticky floor, Gender. 


\section{INTRODUCCIÓN}

El concepto techo de cristal se empezó a aplicar profusamente en la década de 1980, para dar cuenta de la escasa o nula presencia de mujeres en las cúpulas de las organizaciones, convirtiéndose en un importante constructo para estudiar y entender la fuerza laboral norteamericana. La expresión es utilizada para denominar el conjunto de barreras invisibles que evitan o frenan el acceso de las mujeres a puestos de poder, prestigio o salario elevado en cualquier país (Guil Bozal, 2008). Empero, a pesar de la popularidad adquirida por el término en Estados Unidos y en países de Europa, no ha ocurrido lo mismo en el contexto mexicano, en el cual prácticamente es desconocido o ignorado en amplios sectores de la población.

La poca participación de las mujeres en ámbitos ajenos al hogar y al trabajo doméstico ha sido normalizada; este fenómeno se observa no solo en regiones rurales sino también en las grandes ciudades, incluso en los centros de creación de conocimiento, como lo son las instituciones de educación superior, por antonomasia, espacios en donde se gesta y se fortalece la igualdad y se pugna por extinguir la discriminación y la injusticia. A partir de estas premisas, se realizó el presente trabajo, que tiene por objetivo general contribuir a la visibilización de las relaciones asimétricas de género que prevalecen entre las académicas y académicos en las Instituciones de Educación Superior (IES), caracterizando los elementos que conforman el techo de cristal, y, como vinculación lógica, el suelo pegajoso.

Con base en los antecedentes citados, se realizó una revisión de la estructura organizacional de una Universidad del centro de México, identificando a las académicas que ocupan puestos de primer nivel (rectoría y direcciones); asimismo, se hizo un análisis de la relación de mujeres que cuentan con membresía del Sistema Nacional de Investigadores (SNI) y de las que cumplen con el perfil estipulado por el Programa para el Desarrollo Profesional Docente (PRODEP); todo ello con el propósito de delinear el nivel de su presencia en comparación con el de los académicos varones y determinar el techo de cristal en este caso particular.

\section{ALGUNOS CONCEPTOS TEÓRICOS}

En las Instituciones de Educación Superior (IES) suele ignorarse que se dé el fenómeno llamado “techo de cristal", bajo el argumento de que la remuneración recibida por el empleo desempeñado es igual para hombres y mujeres y que su ascenso está regido en condiciones de igualdad. Análisis de casos demuestran, por el contrario, que hay una multiplicidad de hechos que desmentirían este razonamiento, como el otorgamiento de horas extras y las gratificaciones, entre otros; con una franca proporción favorable hacia los varones. En cuanto a la posición laboral de las mujeres, Matus \& Gallego (2015: 612), afirman tajantemente que: "Las estadísticas de mercado de trabajo son concluyentes. Las mujeres 
escasamente acceden a las categorías profesionales más altas.” Ese es el llamado techo de cristal: un conjunto de mecanismos sutiles que impiden que las mujeres lleguen, con igual frecuencia que los hombres, a puestos altos de toma de decisiones. Barberá y cols. (2002: 58) lo definen como una "metáfora [con la que] se pretende representar, de una manera muy plástica y elocuente, las sutiles modalidades de actuación de algunos mecanismos discriminatorios [que] obstaculizan el desarrollo profesional de las mujeres, las limitan y les marcan un tope difícil de sobrepasar”.

Como en las organizaciones laborales, la desigualdad entre hombres y mujeres se revela de manera más que evidente en la academia, sólo que la normalización de esta desigualdad hace que sea poco percibida entre los mismos profesores/as, como lo denuncian Matus \& Gallego (2015: 611).

La carrera académica en la educación superior no es el mejor ejemplo de igualdad [...] En otros sectores [...] los techos de cristal han ido disminuyendo [...] en la universidad prácticamente no se han producido modificaciones. Pese a ello, la percepción del problema es baja en el profesorado.

El techo de cristal, como conjunto de obstáculos que impiden el acceso [o la promoción -ascensoo la permanencia] de las mujeres a y en los puestos altos de trabajo, es invisible, pero sólido. La invisibilidad impide hacer explícitos los mecanismos de sujeción para las mujeres y, más aún, no permite ver elementos discriminatorios contra ellas.

Las conclusiones de Guil (2008), citado por Matus \& Gallego (2015: 614), son más que enfáticas: "Una revisión de las estadísticas de participación de mujeres y hombres [...] muestra que se repite un mismo fenómeno: un descenso de la tasa de participación femenina a medida que se incrementa el nivel académico y lo contrario con la masculina."

La metáfora del techo de cristal se ha ido complejizando en la medida en que ha permitido hacer visibles las desigualdades. Es tan complejo que ahora, como afirman Baxter y Wright (2000), citados por Matus \& Gallego (2015: 614):

no parece ser una barrera localizable y fácil de identificar sino más bien un laberinto de cristal compuesto de una serie de caminos que no conducen a la cima organizacional (Berenguer et al., 1999). Además, y en esta misma línea, el TC se comporta como un tipo de mochila que va acumulando los obstáculos a lo largo de la vida laboral. Se une así este fenómeno al de los suelos pegajosos, vale decir, la sobre representación de las mujeres en la parte baja de la pirámide organizacional.

La relación desigual y altamente discriminatoria para las mujeres ha sido tal, que la denominación de techo de cristal se vuelve, en la práctica, un techo de cemento, al que apenas unas cuantas mujeres han visto o tocado levemente, sin la frecuencia ni intensidad necesarias para, siquiera, provocar una ranura perdurable.

Por otra parte, diversos trabajos de investigación sobre el género en la educación y, sobre todo, acerca del techo de cristal y el suelo pegajoso, son evidencia de que: "Incorporar la perspectiva de género 
en el análisis organizacional, aunque sea necesario, no resulta una tarea fácil," como lo asientan Barberá y colaboradores en su artículo multicitado Más allá del techo de cristal. Diversidad de género (2002: 55). La visibilización de este techo transparente puede ser un buen comienzo a la que aspiramos a contribuir con este trabajo.

\section{RUTA METODOLÓGICA}

La investigación es de carácter documental, vista como procedimiento científico de indagación, recolección, organización, análisis e interpretación de información en torno al tema de techo de cristal en la IES que es motivo de análisis y que identificaremos como "Universidad"; la información estadística está extraída de su página oficial y de los sitios del CONACyT y de la SEP-PRODEP.

\section{RESULTADOS. LA CIMA Y LOS COMPONENTES DEL TECHO DE CRISTAL}

La Universidad tiene una estructura conformada por la rectoría general, las rectorías de los cuatro campus y la dirección del Colegio del Nivel Medio Superior (integrado por once escuelas preparatorias o de bachillerato -nivel preuniversitario-). Por lo que toca al personal académico, como se verá detalladamente, mientras más altos son los niveles de reconocimiento SNI o el número de académicas (os) con perfil PRODEP, existe una menor presencia de mujeres.

\subsection{COMPOSICIÓN DE LA ESTRUCTURA ORGANIZACIONAL DE LA UNIVERSIDAD}

La Universidad tiene un Rector General (nivel más alto). En el segundo nivel están las rectorías de campus: de las cuatro, dos son ocupadas por mujeres; los rectores de los otros dos campus, así como el director general de las 11 escuelas de bachillerato, integran el resto de las primeras cabezas. Es decir, se ubican dos mujeres de seis posibles en estos primeros niveles.

\section{Estructura organizacional del Campus 1}

El campus está presidido por una mujer. En cuanto a las divisiones (facultades) que lo conforman, el de Ciencias de la Salud e Ingenierías es dirigida por una mujer y el de Ciencias Sociales y Administrativas, por un hombre.

\section{Estructura organizacional del Campus 2}

El campus 2 está encabezado también por una rectora; cinco de sus seis divisiones las dirigen hombres y sólo la que se dedica al área de las ciencias económicas y administrativas tiene una directora.

\section{Estructura organizacional del Campus 3}

El campus está rectorado por un hombre. Sus dos divisiones, dedicadas al estudio de las ciencias de la vida y a las ingenierías, son dirigidas por varones. 


\section{Estructura organizacional del Campus 4}

El campus está bajo el cargo de un hombre, así como las tres divisiones que lo integran: ingenierías, ciencias de la salud y ciencias sociales y humanidades.

En síntesis, en el nivel de direcciones de división o facultad, que son 13, solamente hay dos directoras. Esta desproporción ilustra la asimetría y mueve a la pregunta: si las reglas para optar por ese puesto no distinguen entre hombres y mujeres, porque éstas son una franca minoría. Parte de la respuesta proviene del método de designación de estas autoridades, que, en el caso de la Universidad, corresponde a la Junta de Gobierno, organismo conformado por 11 integrantes: ocho son académicos/as de la misma institución y tres más son externos (general, pero no exclusivamente académicos de otras IES). Pues bien, esta Junta nunca ha tenido más de tres mujeres a la vez en sus sucesivas conformaciones, siendo lo normal que sean dos o tres. Si el número de hombres y mujeres del profesorado que pueden formar parte de este órgano tan importante no varía sustancialmente, cabe perfectamente la pregunta porqué desde aquí disminuye tanto el número de ellas. Parte del techo de cristal está aquí: la designación de las autoridades unipersonales pasa por el tamiz, la mirada y la cosmovisión mayoritariamente masculina y designan, en consecuencia, a varones.

Sin embargo, desde antes de llegar a la Junta, el número de aspirantes y candidatos a ocupar estos puestos es, con mucho, muy superior al de las aspirantes mujeres. Las académicas se animan a participar muy poco y se registran en poca proporción, aun siendo una convocatoria abierta y contando con los requisitos estipulados. Otra parte de la respuesta a qué compone el techo de cristal puede residir, entonces, en la escasa participación política de las mujeres (porque estos puestos de índole académica tienen un gran ingrediente político); recordemos que el ámbito político-público ha estado, históricamente, vedado para ellas y es parte de la raíz machista que se reproduce aun en el ámbito académico.

\subsection{BALANCE DE INVESTIGADORAS/ES CON RECONOCIMIENTO EN EL SISTEMA NACIONAL DE INVESTIGADORES Y CON PERFIL PRODEP}

Una actualización de la Universidad respecto a los y las académicas con SNI fue el 29 de marzo de 2017, contando con 480 miembros, es decir, que hubo un aumento de casi el $84 \%$ en comparación con el 2011. De éstos, 157 son mujeres y 323 hombres. ${ }^{1}$. La categoría de emérito o el más alto reconocimiento, lo ostenta un hombre. En el siguiente nivel, el 3, sólo hay una investigadora. La relevancia de pertenecer al Sistema no sólo es simbólica, sino que representa una gratificación económica, cuyo monto varía considerablemente entre el nivel inicial o Candidato, hasta el más prestigiado o Emérito. Una pronta

\footnotetext{
${ }^{1}$ El dato anunciado el pasado 20 de noviembre reportaba que la Universidad cuenta con 590 investigadores en el Sistema Nacional de Investigadores, pero no fue posible hacer el análisis disgregado.
} 
lectura nos conduce también a inferir que las mujeres enfrentan esta siguiente desigualdad. Por tanto, si bien ser investigador SNI no está relacionado con ningún puesto de trabajo en la Institución, sí que otorga poder: reconocimiento en la propia IES y en el claustro de profesores, acceso a apoyos institucionales que están vedados para quienes no tienen esta membresía, liderazgo en torno a los proyectos de investigación y docencia, y, por supuesto, a la gratificación económica.

El componente del techo de cristal lo vinculamos en este caso con las áreas del conocimiento, pues los profesores que tienen las categorías más altas provienen, prácticamente en exclusiva, de las áreas de las ciencias naturales y exactas y de las ingenierías. Lo anterior nos recuerda la tipificación de los campos del conocimiento propios de hombres y de mujeres: para ellos, los de "inteligencia"; para ellas, los de "asistencia". En resumen, la feminización de ciertas áreas académicas trae lastres como los señalados: menor valoración, menor reconocimiento, menor percepción económica.

Por otra parte, 528 de los académicos y académicas de la UG cuentan con perfil PRODEP, ${ }^{2}$ de los cuales 445 tienen el grado de Doctorado, 71 de Maestría y 3 de Especialidad. La adscripción por Campus es como sigue.

\section{Campus 1}

En el campus se encuentran las Divisiones de Ciencias de la Salud e Ingenierías y de Ciencias Sociales y Administrativas. Del total de investigadores en el SNI, el 34.13\% son mujeres y se distribuyen en los departamentos cuyas áreas son la enfermería, los estudios sociales y culturales y la ingeniería agroindustrial. Respecto a las académicas con Perfil PRODEP, se contabilizan 23, siendo este campus un caso excepcional, puesto que hay más mujeres que hombres dentro del PRODEP, diecinueve de ellas con título de doctorado. Esta proporción más equilibrada en cuanto a la relación hombres-mujeres, se explica, entre otros factores, por la presencia tan notable de la enfermería, tradicionalmente confinada como carrera y ejercicio profesional feminizado. Si bien hay presencia de los estudios de ingeniería, esta es del área agroindustrial (no civil, mecánica, eléctrica...), vinculada más a la alimentación, tarea inherentemente relacionada con el mundo femenino.

\section{Campus 2}

Este campus se integra por seis divisiones: Arquitectura, Arte y Diseño; Ciencias Económico Administrativas; Ciencias Naturales y Exactas; Ciencias Sociales y Humanidades; Derecho, Política y Gobierno; e Ingenierías. En este campus, el 31.05\% del total de investigadores SNI son mujeres, con

\footnotetext{
${ }^{2}$ La lista más reciente de profesores y profesoras con perfil PRODEP emitida el 02 de octubre de 2017, daba cuenta de 684 profesoras/s con este reconocimiento.
} 
mayor presencia en Diseño y Farmacia, y en menor proporción en Ingenierías y Astronomía; en este último departamento, no existía ninguna mujer académica como profesora de tiempo completo. En contraparte, 86 académicas de este campus tienen perfil PRODEP.

\section{Campus 3}

El campus se conforma por dos Divisiones: Ciencias de la Vida e Ingenierías. Por razones vinculantes con el área de las ingenierías “duras”, se puede explicar que sólo el 19.35\% de los miembros del SNI son mujeres, ninguna de ellas en ingeniería eléctrica ni mecánica. El dato ratifica las secuelas de una tradición profundamente arraigada aún en determinados contextos socioculturales: la reserva de campos del conocimiento y de ejercicio profesional casi en exclusiva para los varones, en este caso, las ingenierías eléctrica, electrónica y mecánica. En el análisis del Perfil PRODEP, únicamente 22 de ellas tenían ese reconocimiento. Un punto de interés es la ausencia de mujeres con este reconocimiento en los Departamentos de Ingeniería Eléctrica y de Ciencias ambientales.

\section{Campus 4}

En el campus 4 se encuentran tres divisiones (Ciencias de la salud, Ciencias e Ingenierías y Ciencias Sociales y Humanidades). Un 36.28\% de miembros del SNI son mujeres, ninguna de ellas presente en enfermería y bajamente representadas en las demás áreas. Respecto al perfil PRODEP, 40 de los 100 docentes son mujeres, mayormente concentradas en Ciencias de la salud aplicadas al trabajo y menormente representadas en Enfermería y Física.

Como lo anticipamos, ser miembro del Sistema Nacional de Investigadores o contar con el reconocimiento del Perfil PRODEP no se relaciona con los puestos de trabajo ocupados en la estructura organizacional de la Universidad; pero sí se traducen en mejores posiciones en un escalafón imaginario pero igualmente presente, que facilita la participación del profesorado en actividades, comisiones y encomiendas que ponen como requisito ostentar las distinciones anteriores; tienen mucho peso también en el reglamento de evaluación interna del desempeño para el otorgamiento de gratificación económica. Es decir, por sí solos, el SNI ni el PRODEP son puestos en el escalafón, pero sí son medios para ello. El techo de cristal en este caso suma otro ingrediente: para acceder al Sistema o al PRODEP, es necesario contar con el grado mínimo de maestría (para el SNI, prácticamente es indispensable ser doctor), y aunque las estadísticas muestran un abatimiento de la brecha mujeres-hombres, aún es notoria la diferencia a favor de ellos. El suelo pegajoso se hace visible cuando ellas optan por seguir o bien su curso reproductivo o bien la carrera productiva, en términos académicos. 


\section{CONCLUSIONES}

En el análisis de la estructura organizacional de la Universidad y la revisión de la presencia de las académicas en el SNI y con perfil PRODEP, se pueden observar tendencias mencionadas por especialistas en el tema, por ejemplo, la mayoría de las mujeres se concentran en carreras de tipo asistencialista y, a pesar de ello, los hombres tienen mayor reconocimiento en estos campos. Así también, existe menor presencia femenina en Ingenierías, Tecnología y Matemáticas, específicamente en Ingeniería Eléctrica y en Astronomía; no obstante, resalta que tampoco se encuentran mayoritariamente mujeres en Enfermería y obstetricia, profesión que puede considerarse asistencialista, lo que merece considerar que la labor en sí misma no es apreciada por los parámetros de evaluación del SNI y del PRODEP.

En contraparte, y de manera creciente, se manifiesta el ingreso de académicas a departamentos "masculinizados" como algunas ingenierías, ciencias exactas y naturales.

En síntesis, se puede concluir que:

- La estructura organizacional de la Universidad está copada por hombres. En el segundo nivel hay dos mujeres; en el tercero, sólo dos de trece posibles.

- Pudo observarse un techo de cristal en la universidad que propicia desigualdad entre los académicos, especialmente en las áreas de ingeniería y ciencias exactas, de igual manera en niveles elevados de la jerarquía institucional.

- El leaky pipeline dentro de la Universidad es visible a la comunidad universitaria pero es invisibilizado por ciertos mecanismos dentro de la misma; la poca presencia de representantes femeninas en los altos mandos de la institución es alarmante y es un punto que debe ser abordado con prontitud por parte de las personas que toman decisiones, animadas por una comunidad que se presume crítica.

- La representación femenina en departamentos considerados socialmente masculinos empieza a hacerse visible, lo cual indica un avance hacia la igualdad, pero un análisis detallado, revela que tomará años antes de ver una relación más asimétrica y que tampoco significa que las mujeres ocupen sitios de primer nivel.

- Los órganos de gobierno tienen una evidente presencia de hombres. Además, son las autoridades unipersonales las que los presiden: la toma de decisiones, que marca el destino de la universidad, es ejercida por una marcada mayoría masculina.

- Finalmente, la elección de autoridades unipersonales recae en la figura de la Junta Directiva, compuesto por once integrantes. Nunca, en sus años de existencia, ha habido cuatro mujeres a la vez. La mano que mece la cuna es también, masculina. 


\section{REFERENCIAS}

Audelo, C. B., Rodríguez, M. S. \& Urrea, M. L. (septiembre, 2009). La segregación académico/laboral de las profesoras de la Universidad Autónoma de Sinaloa. Ponencia presentada en el X Congreso Nacional de Investigación Educativa. Veracruz, México.

Barberá, E., Ramos, A., Sarrió, M. \& Candela, C. (2002). Más allá del techo de cristal. Diversidad sexual [versión electrónica]. Revista del Ministerio de Trabajo y asuntos sociales. Núm. 40. España.

Garza, B. (2017). "Mujeres y liderazgo: desafíos para acceder a puestos directivos en educación superior". Ponencia presentada en el XIV Congreso Nacional de Investigación Educativa. COMIE/Universidad de San Luis Potosí: México.

González, A. (2015). El techo de cristal. Tesis para obtener el Máster Universitario en Dirección y Administración de Empresas. Universidad de Oviedo. En: http://digibuo.uniovi.es/dspace/bitstream/10651/33742/3/TFM_GonzalezMartinez\%2C\%20Ana.pdf

Guil, A. (2008). Mujeres y ciencia: techos de cristal. EccoS Revista Científica, 10(1), 213-232. Recuperado de: http://www.redalyc.org/articulo.oa?id=71510111

Matus-López, M. \& Gallego-Morón, N. (2015). Techo de cristal en la universidad. Si no lo veo no lo creo. Recuperado el 13 de febrero de 2017, https://www.researchgate.net/publication/283004601_Techo_de_cristal_en_la_Universidad_Si_no_lo_v eo_no_lo_creo.

Padilla, L., Scott, A. \& Galaz-Fonte, J. F. (2011). Las mujeres académicas y el techo de cristal. Una perspectiva comparada en Norteamérica (México, Estados Unidos y Canadá). Ponencia presentada en el XI Congreso Nacional de Investigación Educativa. Distrito Federal, México.

Surovikina, E. (2017). Gestión educativa desde la perspectiva de género: un panorama integrado a través del lenguaje. Ponencia presentada en el XIV Congreso Nacional de

Viloria, E. \& Santillán, V. E. (2013). Trayectorias académicas y el techo de cristal en Derecho y Economía. Ponencia presentada en el XII Congreso Nacional de Investigación Educativa. Guanajuato, México. 